\title{
Overexpression of HSP27 in cultured human aortic smooth muscular cells reduces apoptosis induced by low-frequency and low-energy ultrasound by inhibition of an intrinsic pathway
}

\author{
B. Zhang ${ }^{1,2}$, H.S. Zhou ${ }^{3}$, Q. Cheng ${ }^{4}$, L. Lei ${ }^{2}$ and B. Hu ${ }^{1}$ \\ ${ }^{1}$ Shanghai Jiao Tong University, Affiliated 6th People's Hospital, \\ Department of Ultrasound in Medicine, \\ Shanghai Institute of Ultrasound in Medicine, Shanghai, China \\ ${ }^{2}$ Affiliated Shanghai East Hospital, Department of Ultrasound in Medicine, \\ Tongji University, Shanghai, China \\ ${ }^{3}$ Shanghai Acoustics Laboratory, Chinese Academy of Sciences, \\ Shanghai, China \\ ${ }^{4}$ Institute of Acoustics, Tongji University, Shanghai, China
}

Corresponding author: B. Hu

E-mail: binghubo@yahoo.com.cn

Genet. Mol. Res. 12 (4): 6588-6601 (2013)

Received June 21, 2013

Accepted October 2, 2013

Published December 11, 2013

DOI http://dx.doi.org/10.4238/2013.December.11.10

\begin{abstract}
We investigated in vitro the effect of low-frequency and low-energy ultrasound (LFLEU) on apoptosis of an overexpressed HSP27 human aortic smooth muscle cell (HASMC) line. A frequency of $42.6 \mathrm{kHz}$ was used in all experiments. HASMC were exposed to ultrasound and cell viability was evaluated by MTT reduction. Overexpressed HSP27-HASMC was constructed on a pcDNA3.1 vector. Apoptosis was determined $24 \mathrm{~h}$ after treatment by flow cytometry; gene display was evaluated with Affimax chips, and HSP27 mRNA and protein expression levels were measured by RT-PCR and Western blotting. The apoptosis rate (at $30 \mathrm{~s}$ ) was significantly lower in
\end{abstract}


HASMC transfected with HSP27 (7.14 $\pm 1.73 \%)$, compared with cells transfected with a mock plasmid $(17.31 \pm 2.72 \%)$ or a control group $(14.23 \pm 2.77 \%)$, indicating a protective function for apoptosis induced by LFLEU. Gene display analysis showed that caspase-9 expression in HSP27 cell lines was downregulated and caspase-3 upregulated. However, RT-PCR and Western blotting analysis indicated that both caspase- 9 and caspase- 3 were inhibited at both the mRNA and protein levels. We suggest that overexpressed HSP27 is capable of protecting the LFLEU from apoptosis and that the pathway for this protection is via downregulated caspase-9 and caspase-3 expression.

Key words: HSP27; Human aortic smooth muscle cell; Ultrasound; Overexpression

\section{INTRODUCTION}

Although biological effects of ultrasound have been studied and the clinical use of ultrasound is well documented, the exact cellular and molecular effects of ultrasound are still relatively little known, particularly for low-frequency ultrasound (Baker and Dalrymple, 1978; Palumbo et al., 2011). Apoptosis, or programmed cell death, is a normal physiologic process that contributes to the homeostasis of tissues and maintains the balance between cell proliferation and cell death. Apoptosis is always a special cellular response to stress stimuli, such as exposure to chemotherapeutic drugs, oxidative stress, free radicals, X-rays, ultraviolet radiation, and physical stress including ultrasonic irradiation (Feng et al., 2008).

Many studies have suggested that cell apoptosis could be induced by low-intensity ultrasound (10-60 kHz) or high-intensity ultrasound (Lagneaux et al., 2002; Firestein et al., 2003; Fang et al., 2007; Feng et al., 2008; Tsuang et al., 2011) (700 kHz to several megahertz). Recently, low-frequency $(10-60 \mathrm{kHz})$ ultrasound for enhancement of various cellular signal processes has attracted much attention as a rapid method (Lagneaux et al., 2002; Rokhina et al., 2009).

Low-frequency ultrasound $(20-100 \mathrm{kHz})$ has a diverse set of industrial and medical applications. In fact, high-power industrial applications of ultrasound mainly occupy this frequency range. This range is also used for various therapeutic medical applications including sonophoresis (ultrasonic transdermal drug delivery), dentistry, eye surgery, body contouring, breakage of kidney stones, and elimination of blood clots (Ahmadi et al., 2012). Low-frequency ultrasound irradiation under a certain acoustic intensity can increase blood-brain barrier permeability non-invasively and reversibly (Xia et al., 2012). Low-frequency ultrasound $(45 \mathrm{kHz})$ is able to influence the proliferation and differentiation of odontoblast-like cells and may potentially be considered as a therapeutic tool for dental pulp and dentine repair (Man et al., 2012).

Over the past two decades, there has been an increasing interest in the relationship between Hsp27 and cardiovascular disease. Heat shock proteins (HSP), which are induced in response to stress such as heat and chemical stress, act as molecular chaperones, which contribute to various effects, including protein folding, oligomerization, and protection of cells from apoptosis (Hendrick and Hartl, 1993). The mechanism for this protection remains 
unclear. HSP are generally classified into two categories, high molecular weight HSPs and low molecular weight HSPs (small HSPs) (Liu et al., 1989; Taylor and Benjamin, 2005). Although the functions of small HSPs with a molecular mass from 10 to $30 \mathrm{kDa}$, such as HSP27, have not been thoroughly investigated, accumulating reports regarding the small HSPs have shown that they have causative or preventive effects for various human diseases (van Noort et al., 1995; Martin et al., 1997; Mackay et al., 2003; Meehan et al., 2004; Chelouche-Lev et al., 2004). For example, overexpression of HSP27 and B-crystallin protects heart cells against ischemia/reperfusion injury, similar to HSP70 (Hollander et al., 2004). HSPs (HSP90, HSP84, HSP70, HSP27, HSP20, and alpha B crystallin) all appear to play a significant cardiovascular role (Benjamin and McMillan, 1998). HSP27 is a major phosphoprotein during muscle contraction. HSP27 functions in smooth muscle migration and appears to serve an integral role (Salinthone et al., 2008; Kampinga et al., 2009). Vascular smooth muscle cell (VSMC) proliferation plays an important role in the pathologic restenosis of blood vessels after percutaneous transluminal angioplasty and stenting (Reimers et al., 1997). Arakawa et al. (2002) reported that sonodynamic therapy could reduce intimal hyperplasia after stenting. However, the frequencies of ultrasound for inhibiting the proliferation of VSMCs usually exceed $650 \mathrm{kHz}$. We do not know if HSP27 plays any protective role in smooth muscle cell apoptosis. So far, there are still few reports about the effect of irradiation of low-frequency and low-energy ultrasound (LFLEU) on apoptosis of VSMCs and the apoptotic pathway induced by low-frequency ultrasound remains unclear.

The present study was performed to examine the effect of different ultrasonic treatment conditions on an HSP27-overexpressing human aortic smooth muscle cell (HASMC) line (HSP27-HASMC) and to investigate the possible role of HSP27 overexpression in apoptosis induced by LFLEU treatment. The experiments were performed with flow cytometry (FACS) and gene display to investigate the possible protective mechanism of apoptosis induced by LFLEU treatment. The results confirmed by Western blotting and RT-PCR suggest that overexpressed HSP27 in HASMC is capable of protecting from apoptosis induced by low-frequency ultrasound, and the possible mechanism for this protection is inhibiting caspase- 9 and caspase-3 expression at both the mRNA and protein level.

\section{MATERIAL AND METHODS}

\section{HASMC}

HASMC were purchased from Shanghai Institute of Cell Biology and cultured in 100 x 20-mm tissue culture dishes (Falcon, Oxnard, CA, USA) in Dulbecco's modified Eagle's medium (DMEM) supplemented with 5\% fetal bovine serum and 5\% newborn calf serum, $2 \mathrm{mM}$ L-glutamine, $100 \mathrm{U} / \mathrm{mL}$ penicillin, $100 \mu \mathrm{g} / \mathrm{mL}$ streptomycin (Hyclone) at $37^{\circ} \mathrm{C}$ in a humidified atmosphere of 5\% $\mathrm{CO}_{2}$ and $95 \%$ air. The cells were subcultured twice a week and cells were counted in a hemocytometer (Anxin Optical Equipment, Shanghai, China). The viability of the cells was determined by the trypan blue exclusion test. In all cases, viability was greater than $90 \%$. Cultures were passaged upon reaching confluence by brief exposure to PBS containing $0.5 \mathrm{mg} / \mathrm{mL}$ trypsin and $0.5 \mathrm{mM}$ EDTA at $37^{\circ} \mathrm{C}$, and subcultured in DMEM supplemented with serum and antibiotics as described above. Cell growth was observed under an inverted phase contrast microscope (Carl Zeiss, Oberkochen, Germany). 


\section{Ultrasound apparatus}

The ultrasonic transducer units operating at a frequency of $42.6 \mathrm{kHz}$ (effective radiating area $38.04 \mathrm{~cm}^{2}$ ) were positioned sidewise at the bottom of the water bath. The transducer was connected to an ultrasound wave generator (model: DM-40; Shanghai Acoustics Laboratory, Chinese Academy of Sciences), which worked in a continuously adjustable frequency and its maximum output electric power was about $50 \mathrm{~W}$. Also, the output electric power was monitored by a digital electric power analyzer (model: PPA2500; N4L).

The tubes containing the cell suspension were positioned in the tube-holder so that the cell suspension was in the middle of the transducer at a distance of $10 \mathrm{~mm}$ from its surface within the "near field" of the ultrasound beam (Figure 1). The tubes were made of polystyrene. A needle-type hydrophone (model: SPRH-S-1000; SEA), connected to a digital oscilloscope (model: TDS 1024B; Tektronix), was used to check the operating conditions.

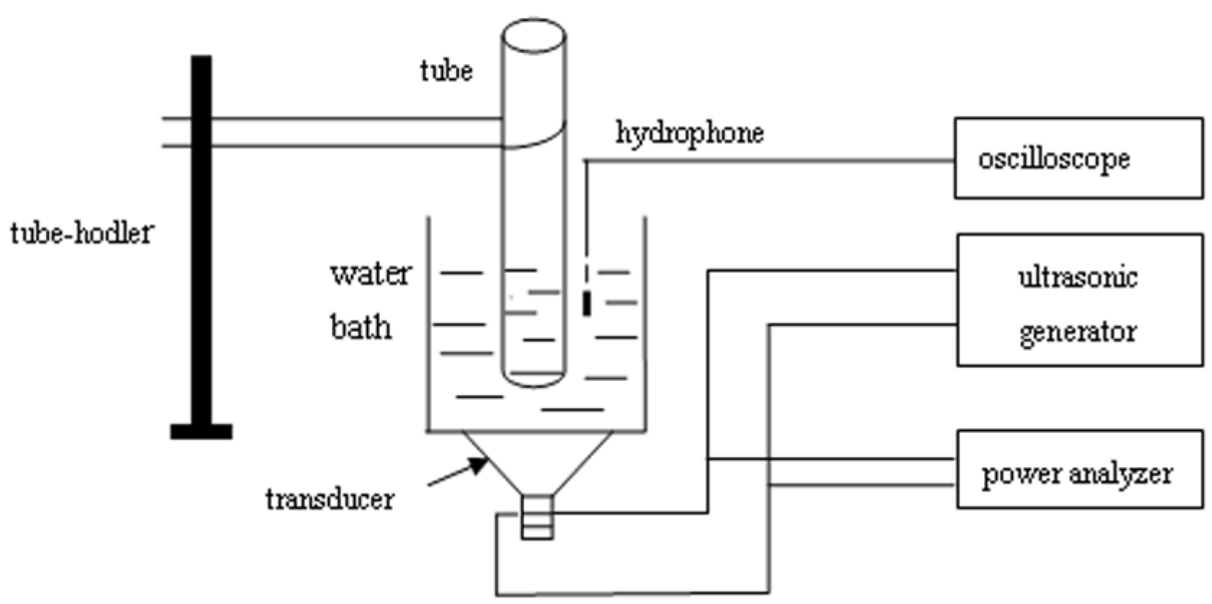

Figure 1. Schematic diagram of sonication set-up.

\section{Protocol for ultrasound treatment}

HASMC of confluent cultures were trypsinized and then well suspended: $5 \times 10^{5} / \mathrm{mL}$ DMEM in a culture tube measuring $16 \times 125 \mathrm{~mm}$ (Corning, Staffordshire, UK). They were then exposed to ultrasound using a sonicator (model: DM-40 Shanghai Acoustics Laboratory, CAS). The experimental system mainly consisted of two parts: an ultrasonic generator (DM-40) and a transducer with container that operated at $42.6 \mathrm{kHz}$. During the experiment, we adjusted the output power of the ultrasonic generator to reach cavitation threshold, and a needle-type hydrophone (model: SPRH-S-1000; SEA) was used to measure ultrasonic intensity. Moreover, due to the thermal effect of ultrasound, the temperature in the culture tube could increase, so to avoid the effect of temperature on the experimental results, we put the culture tube in a thermostatic water bath (precision: $\pm 0.05^{\circ} \mathrm{C}$ ), and the temperature was measured by a thermocouple in the test tube and the bath. The time of exposure to ultrasound was set at 0 , $10,20,30,35$, and $40 \mathrm{~s}$ for each tube of cells. 


\section{Viability assessment of sonicated HASMC by methylthiazol tetrazolium (MTT) assay}

In the MTT assay, aliquots of HASMC (at 10,000 cells per well) were trypsinized, well suspended, sonicated, and then transferred to a fibronectin $\left(25 \mathrm{ng} / \mathrm{mm}^{2}\right)$-precoated flatbottomed 96 -well microplate $(0.1 \mathrm{~mL} /$ well $)$. Briefly, the MTT assay was performed according to manufacturer recommendations, as described before. The absorption was determined at $560 \mathrm{~nm}$ using a microplate reader (Kontron SLT-210-Thermo Fisher Scientific, $\mathrm{CO}$, USA). Tetrazolium dissolved in DMSO and diluted in PBS to a final concentration of $7 \mathrm{mM}$ was added (10 $\mu \mathrm{L} \mathrm{MTT/well)} \mathrm{to} \mathrm{each} \mathrm{well.} \mathrm{After} \mathrm{incubation} \mathrm{for} 4 \mathrm{~h}, 100 \mu \mathrm{L} 0.004 \mathrm{~N}$ $\mathrm{HCl}$-isopropanol was pipetted into each well and the contents vigorously mixed by repeated pipetting. After 5 min, the plates were read with an ELISA reader (Kontron SLT-210) at a wavelength of $492 \mathrm{~nm}$ and the results expressed as OD (optical density). The stimulation index is defined as the ratio of the mean OD of sonicated SMC to the mean OD of nonsonicated, nonstimulated cells. Nonsonicated cells served as the control. A standard curve indicating the OD signal $v s$ the number of cells was performed by serial dilutions of quantitated HASMC measured by the MTT method.

\section{Cloning of stable HSP27-overexpressing cell line}

\section{Construction of pcDNA3.1-Hsp27 vector}

PCR was used to amplify the HSP27 fragment of the pMD-HSP27 vector, obtained from CLONTECH Laboratories (Clontech, Mountain View, CA, USA). The 5' primer (5'AAA GAA TTC ATG ACC GAG CGC CGC GTC-3') and the 3' primer (5'-TTT GGA TCC TTA CTT GGC GGC AGT CTC A-3') were engineered with an EcoRI and BamHI restriction site, respectively. A 50- $\mu \mathrm{L}$ amplification reaction containing each primer at $1 \mathrm{mM}, 10 \mu \mathrm{g}$ pMD-HSP27 vector, and $1 \mathrm{U}$ Pfu DNA polymerase (TIANGEN, China) was subjected to 30 amplification cycles at $95^{\circ} \mathrm{C}$ for $1 \mathrm{~min}, 60^{\circ} \mathrm{C}$ for $30 \mathrm{~s}$, and $72^{\circ} \mathrm{C}$ for $1 \mathrm{~min} 30 \mathrm{~s}$. The $618-\mathrm{bp} \mathrm{am}-$ plification product was added adenylate at the 3 '-end by Taq DNA polymerase, and the fragment was cloned to the pMD18-T vector, named pMD18-T-HSP27. The pMD18-T-HSP27 was digested with EcoRI to get the HSP27 fragment, and then cloned to the pcDNA3.1-puro vector, which was digested with $X b a \mathrm{I}$ and NheI through flat-end cloning. The resulting plasmid, pcDNA3.1-HSP27, was subjected to sequence analysis to confirm the integrity of the inserted element. The pcDNA3.1-puro-XbaI-NheI fragment was self-ligated as a comparison plasmid, named pcDNA3.1.

\section{Construction of the HASMC-HSP27 cell line}

Determination of antibiotic sensitivity was as follows. Cells were seeded at a density of 250,000 cells/well on 6-well plates and allowed to attach overnight. A range of antibiotic concentrations from 0 to $2.5 \mu \mathrm{g} / \mathrm{mL}$ puromycin were added to the wells. The selective media were replenished every 3-4 days, and the percentage of surviving cells observed. The viable cells were counted at regular intervals to determine the appropriate concentration of puromycin that prevents growth within 2-3 weeks after its addition. 
Lipofectamine $^{\mathrm{TM}} 2000$ (Invitrogen) was used for stable transfection. Cells were plated one day before transfection, and $2 \times 10^{5}$ cells and $2 \mu \mathrm{g}$ plasmid DNA were used for each transfection. After $48 \mathrm{~h}$, the cells were split such that they were no more than $50 \%$ confluent. The cells were fed selective medium every 3-4 days until puromycin-resistant foci could be identified. The puromycin-resistant cells were grown in the well to a certain amount. When the selected monoclonal cells were enough, they were digested with the sterile penicillin papers containing pancreatic enzyme and transferred to a 12-well plate. The monoclonal cells were gradually expanded, resulting in the HASMC-pcDNA3.1-HSP27 and HASMC-pcDNA3.1 cell lines.

\section{Detection of HSP27 expression in the HASMC-pcDNA3.1-HSP27cell line}

Real-time qPCR was used to detect HSP27 expression. The templates were reverse transcription products of the HASMC-pcDNA3.1-HSP27 and HASMC-pcDNA3.1 cell lines, whose RNA was extracted with the RNA extraction kit from TIANGEN, and reversetranscribed using the MBI RevertAid First-Strand cDNA Synthesis kit. The primers of the target gene HSP27 were HSP27-2F (5'-GCC ACG CAG TCC AAC GAG ATC A-3') and HSP27-2R (5'-ACT TGG CGG CAG TCT CAT CGG A-3'); the primers of the control gene GAPDH were GAPDH-F (5'-ACG GAT TTG GTC GTA TTG GG-3') and GAPDH-R (5'CGC TCC TGG AAG ATG GTG AT-3'). According to the TAKARA SYBR Premix Ex TaqII (Tli RNaseH Plus) kit to establish the reaction system, and ABI 7500 Fast Real-Time PCR System to perform PCR, stage 1: initial denaturation, Reps: $1,95^{\circ} \mathrm{C}, 30 \mathrm{~s}$; stage 2 : PCR, Reps: $40,95^{\circ} \mathrm{C}, 3 \mathrm{~s}, 60^{\circ} \mathrm{C}, 30 \mathrm{~s}$; melt curve stage. Amplified products were separated on $1 \%$ agarose gels and photographed with a Kodak Gel Logic 100 Imaging System. The densities of the bands were determined using the Gel-Pro Analyzer 4.0 software and Western blot analysis. HASMC cells were harvested and lysed in nondenaturing lysis buffer (Applygen Technologies, Beijing, China). Samples containing $30 \mu \mathrm{g}$ protein per lane were separated by $10 \%$ sodium dodecyl sulfate-polyacrylamide gel electrophoresis (SDS-PAGE) and transferred to PVDF membranes. The membranes were blocked in 5\% skim milk-TBST (20 mM Tris- $\mathrm{HCl}, \mathrm{pH} 7.5,500 \mathrm{mM} \mathrm{NaCl}, 0.1 \%$ Tween 20 ) for $1 \mathrm{~h}$. The respective primary antibodies were then added in the same milk and the membranes incubated overnight at $4{ }^{\circ} \mathrm{C}$, followed by incubation with horseradish peroxidase-conjugated secondary antibody in TBST for $2 \mathrm{~h}$ at room temperature. The blot was developed with the LAS3000 chemiluminescence system (Fujifilm, Tokyo, Japan).

\section{Detection of apoptotic cells by flow cytometry}

Cells undergoing apoptosis were detected by staining with FITC-conjugated Annexin V and propidium iodide (PI), using the Annexin V-FITC Apoptosis Detection kit and according to manufacturer recommendations (KeyGEN, Nanjing, China). Briefly, the cells were harvested and washed twice with PBS, resuspended in $500 \mu \mathrm{L}$ binding buffer, and then incubated with $5 \mu \mathrm{L}$ Annexin V and $5 \mu \mathrm{L}$ PI for $15 \mathrm{~min}$ in the dark at room temperature. Cells, $1 \times 10^{4}$, were analyzed with a flow cytometer (BD FACSCalibur, USA) by the Cell Quest software, and the percentage of apoptotic cells was determined. 


\section{RT-PCR}

Real-time qPCR was used to detect apoptotic gene expression in HSP27 overexpressing cells. The templates were reverse transcription products of the cell line, whose RNA was extracted with the RNA extraction kit from TIANGEN, and reverse transcribed using the MBI RevertAid First-Strand cDNA Synthesis kit. The primers of the target genes were human caspase-9 F: 5'-TGTTCAGGCCCCATATGAT-3' and caspase-9 R: 5'-CTGGCCTGTGTCCTCTA AGC-3'; human caspase-3 F: 5'-GAGCCATGGTGAAGAAGGAA-3' and caspase-3 R: 5'-GGCAGGCCTGAATAATGAAA-3'. According to the TAKARA SYBR Premix Ex TaqII (Tli RNaseH Plus) kit to establish the reaction system, and ABI 7500 Fast Real-Time PCR System to perform PCR: stage 1: initial denaturation, Reps: $1,95^{\circ} \mathrm{C}, 30 \mathrm{~s}$; stage 2: PCR, Reps: $40,95^{\circ} \mathrm{C}, 3 \mathrm{~s}, 60^{\circ} \mathrm{C}, 30 \mathrm{~s}$; melt curve stage. Amplified products were separated on $1 \%$ agarose gels and photographed with a Kodak Gel Logic 100 Imaging System. The densities of the bands were determined using the Gel-Pro Analyzer 4.0 software.

\section{Western blotting}

Sonicated cells and nonsonicated cells were collected and homogenized, and the homogenate was centrifuged at low speed after $15 \%$ dextran was added. The pellet-containing capillaries were sampled, snap frozen, stored at $-80^{\circ} \mathrm{C}$, and used for Western blotting. Equal amounts of protein $(10-20 \mu \mathrm{g})$ from each group were separated by SDS-PAGE, transferred to a nitrocellulose membrane, and immunostained by monoclonal anti-caspase- 3 and caspase- 9 antibodies (1:1000 dilution). $\beta$-actin stained with polyclonal anti-b-actin (1:300 dilution; Santa Cruz Biotechnology, Santa Cruz, CA, USA) was used as an internal control. Immunoblots were visualized by enhanced chemiluminescence (ECLkit, Santa Cruz Biotechnology). Autoradiographic images were scanned and integrated density values of protein bands were quantified by the Chemi-Imager 5500V2.03 software.

\section{Gene display analysis}

To determine the effect of ultrasound on gene expression in HASMC and HSP27HASMC, the supernatants from cell cultures that were exposed to ultrasound for $30 \mathrm{~s}$ were collected. Invitrogen Trizol was used for extraction of total RNA according to manufacturer instructions. Reverse transcript and cDNA synthesis and biotinylated cDNA from $100 \mathrm{ng}$ total RNA were prepared according to the standard Affymetrix protocols. Briefly, $5.5 \mu \mathrm{g}$ cDNA was hybridized for $16 \mathrm{~h}$ at $45^{\circ} \mathrm{C}$ on GeneChip Human Gene 1.0ST Array. GeneChips were washed and stained in the Affymetrix Fluidics Station 450, and then scanned using the GeneChip Scanner 3000 7G. The data were analyzed with the Partek Genomics Suite version 6.5 using Affymetrix default analysis settings and global scaling as normalization method (Partek Genomics Suite 6.5).

\section{Data acquisition and statistical analysis}

At least three independent experiments were performed per data point. The results are reported as means and standard deviations of these ratios. The differences of the means were analyzed by the Student $t$-test. $\mathrm{P}<0.05$ was considered to be statistically significant. 


\section{RESULTS}

\section{Construction of the HASMC-HSP27 cell line}

The functions of small HSP with a molecular mass from 10 to $30 \mathrm{kDa}$, such as HSP27, have not been thoroughly investigated; accumulating reports regarding small HSP have shown that they have causative or preventive effects for various human diseases (Mackay et al., 2003). To investigate HSP27 functions in HASMC exposed to LFLEU, an HSP27 overexpressing HASMC line was constructed and HSP27 mRNA and protein levels were confirmed by RT-PCR and Western blotting between the pcDNA3.1 vector and higher HSP27 expression groups, as shown in Figure 2.

A

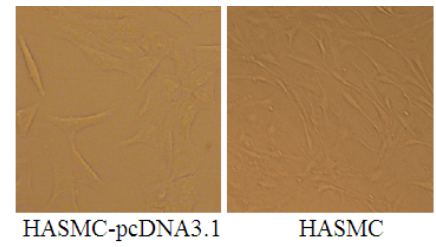

HASMC-pcDNA3.1

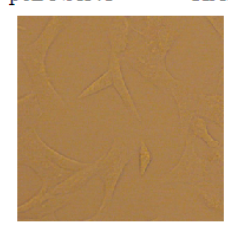

HASMC-pcDNA3.1-HSP27

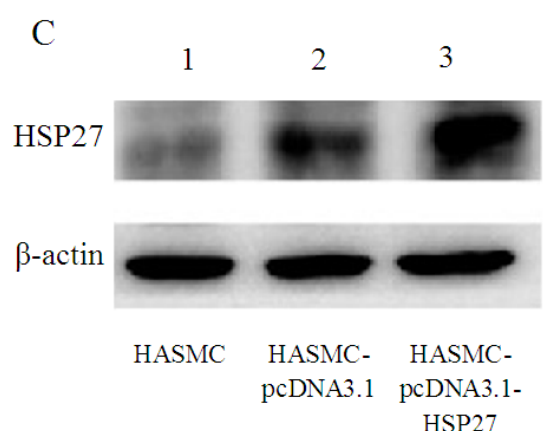

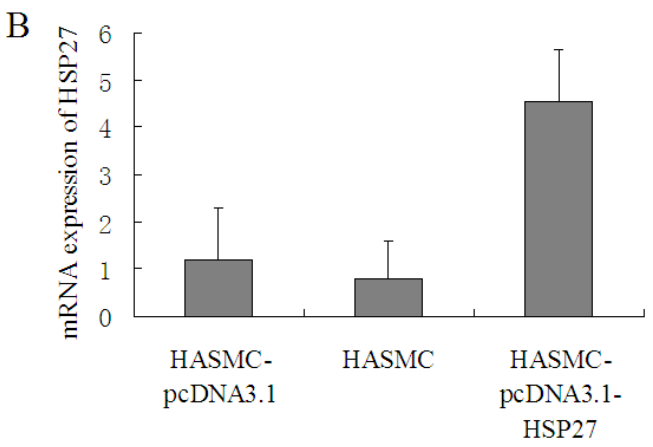

$\mathrm{D}$



Figure 2. Overexpression of HSP27 in human aortic smooth muscle cell (HASMC) line. HASMC were inserted with pcDNA3.1+HSP27 cDNA. Whole cell mRNA and proteins extracted from different groups were subjected to RT-PCR and Western blot analysis. A. HASMC, HASMC-pcDNA3.1, and HASMC-pcDNA3.1-HSP27 cells under microscope (20X original magnifications). B. HSP27 mRNA expression in HASMC. The total RNA was extracted for RT-PCR assay. Raw relative quantitation showed HSP27 mRNA expression in HASMC. C. HSP27 protein expression by Western blot. D. Ratio of HSP27 protein and $\beta$-actin. *P $<0.01$, HASMC-pcDNA3.1-HSP27 vs HASMC-pcDNA3.1; ${ }^{\mathrm{P}}<0.001$, HASMC-pcDNA3.1-HSP27 vs HASMC.

\section{MTT assay to measure changes in cell viability}

The toxicity of ultrasound treatment to HASMC was determined using the MTT 
assay to measure changes in cell viability. The results showed that cell viability decreased with longer exposure time (over $30 \mathrm{~s}$ ), as shown in Figure 3. So we chose $30 \mathrm{~s}$ as the optimal time point for apoptosis analysis by flow cytometry, since at this point there was not much cell death.



Figure 3. Changes in cell viability under ultrasound treatment by MTT assay. The results are reported as means \pm $\mathrm{SD}$ of three individual experiments.

\section{Apoptosis detected by flow cytometry}

Apoptosis was evaluated with flow cytometry to investigate if there was an anti-apoptosis effect by hsp27 overexpression in HASMC. Compared with the control group, the apoptosis rate in the HSP27-overexpressing HASMC line was significantly lower $(7.14 \pm 1.73$ vs $17.31 \pm 2.72 \%, \mathrm{P}<0.01 ; 14.23 \pm 2.77 \%$ vector only, $\mathrm{P}<0.05$, as shown in Figure 4$)$. These results suggested that HSP27 overexpression in HASMC was capable of protecting against apoptosis by downregulation of caspase- 9 and that HSP27 played a very important role in protecting against apoptosis induced by LFLEU.

\section{Gene display analysis}

Under the optimal LFLEU exposure conditions (30 s), HASMC and HSP27-overexpressing HASMC were compared by gene chip analysis. The results showed significant changes in gene expression in caspase signaling pathways. Caspase- 3 was upregulated over 1-fold but caspase- 8 and caspase- 9 were downregulated over 1-fold as shown in Table 1. These results indicate that possible signal pathways for this protection are due to downregulation of caspase-9, which may play key roles in apoptosis induced by low-frequency ultrasound. Considering the gene display signal noise, we used RT-PCR and Western blotting to confirm the results again. 
A

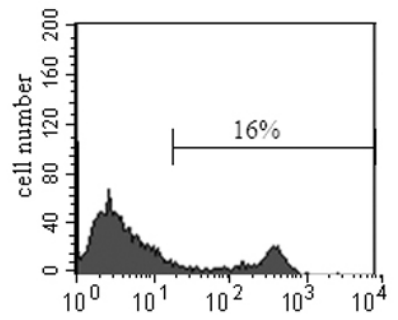

(2)

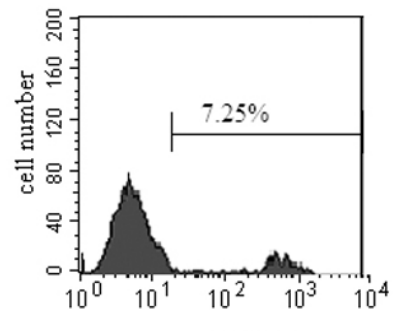

Annexin-V FITC
(3)

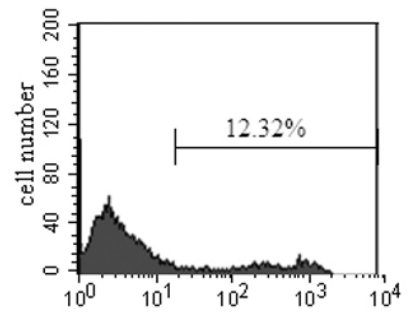

B

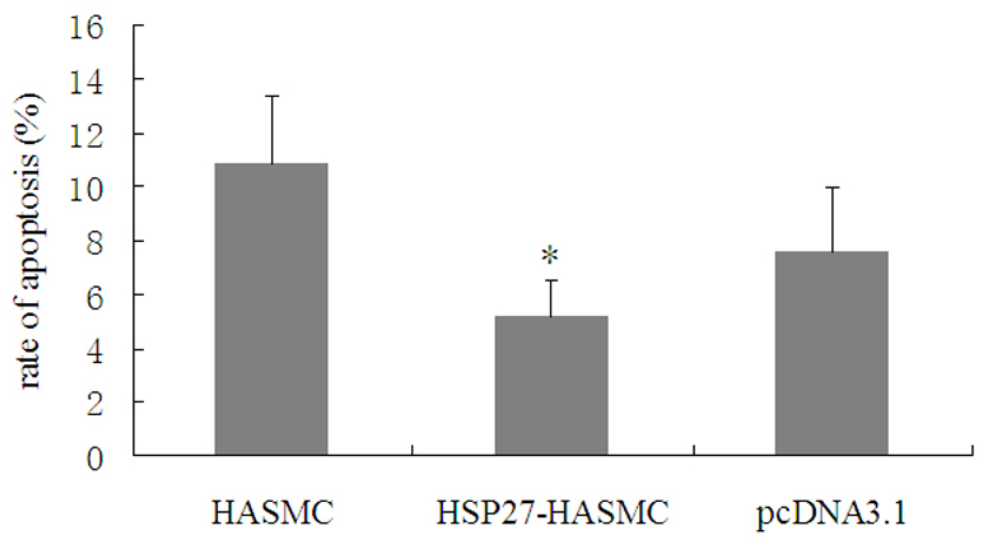

Figure 4. Apoptosis of HSP27-HASMC (human aortic smooth muscle cell) line examined by flow cytometry after exposure of low-frequency and low-energy ultrasound. A. One of individual flow cytometry results from three different groups. (1), (2) and (3) represent HASMC, HSP27-HASMC and pcDNA3.1, respectively. B. Results showing a significant lower rate of apoptosis in the HSP27-HASMC group compared with the control group $(* \mathrm{P}<0.05, \mathrm{~N}=4)$.

Table 1. Differential expressed genes involved in caspase (CASP) signaling pathways.

\begin{tabular}{lc}
\hline Name fold-change (overexpressed HSP27 group $v s$ HASMC group) & \\
\hline CASP8AP2 & 1.45887 up \\
CASP3 & 1.01182 up \\
CASP6 & -1.31528 down \\
CASP8 & -1.13575 down \\
CASP10 & 1.24914 up \\
CASP14 & -1.00248 down \\
CASP1 & 1.43117 up \\
CASP5 & 1.33765 up \\
CASP4 & 1.04137 up \\
CASP12 & -1.15627 down \\
CASP7 & 1.01752 up \\
CASP9 & -1.03515 down \\
\hline
\end{tabular}

HASMC $=$ human aortic smooth muscle cell.

\section{RT-PCR and Western blot analysis of caspase-9 and caspase-3}

RT-PCR and Western blot analysis of caspase-9 and caspase-3 were performed to confirm the results from gene display analysis (Figure 5). The results showed that caspase-9 
and caspase-3 protein levels were downregulated in the sonicated group as shown in Figure 5A and $\mathrm{B}$, which is partly consistent with differential gene display. The expression level of both procaspase-9 (Figure 5B) and procaspase- 3 protein was reduced 52 and $35 \%$, respectively. RTPCR for caspase-9 and caspase-3 mRNA levels in the HSP27-overexpressing group showed a 36 and 29\% reduction, compared with the control group, as shown in Figure 5C. These results confirmed that intrinsic apoptotic pathway played a very important role in cultured rat aortic smooth muscle cells (A7r5 cells) exposed to LFLEU.

A
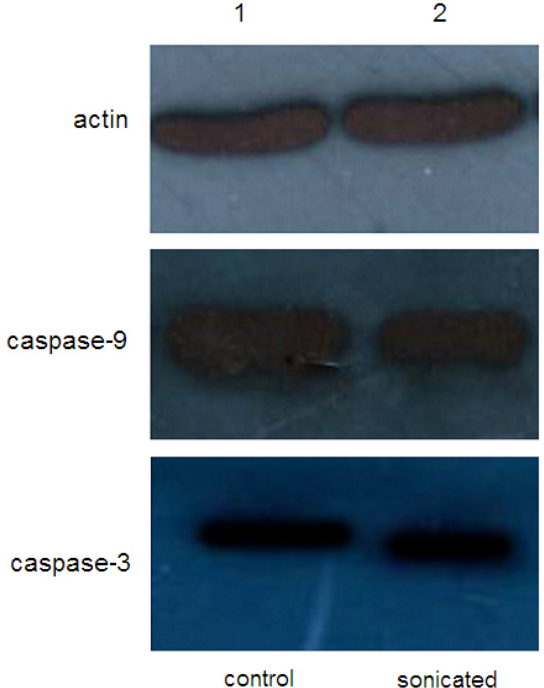

C

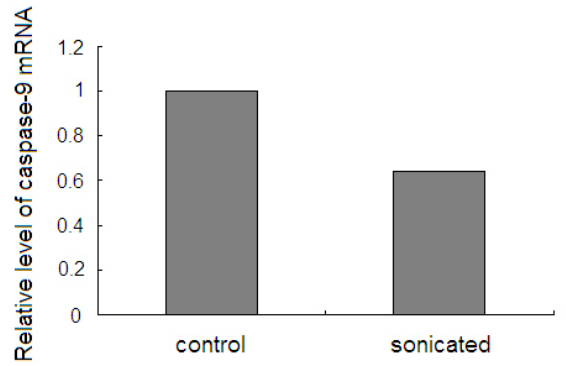

(1)
B

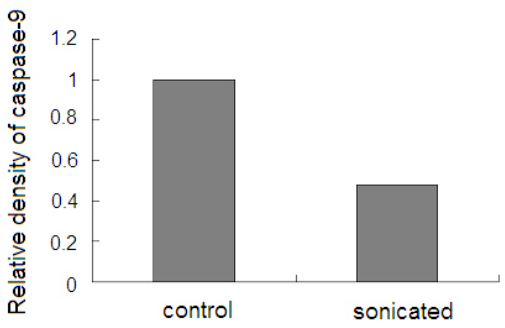

(1)

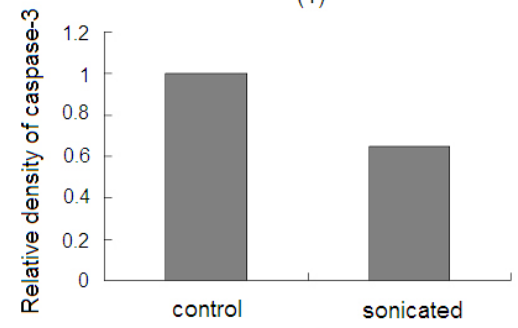

(2)

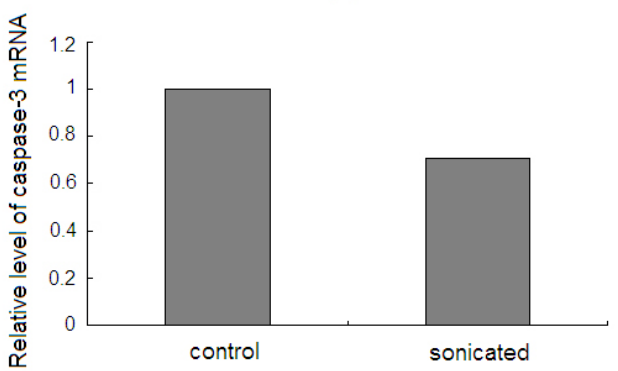

(2)

Figure 5. RT-PCR and Western blot analysis of caspase-9 and caspase-3. A. Immunoblotting assay for the inactive precursor of procaspase-9 and procaspase-3. B. Histograms showing the densitometric analysis of procaspase-9 and procaspase-3, respectively. Expression level of both procaspase-9 and procaspase-3 protein in HSP27 overexpression group had 52 and 35\% reduction, respectively, compared with the control group. C. RT-PCR assay for the inactive precursor of caspase- 9 and caspase- 3 mRNA level. Both caspase- 9 and caspase- 3 mRNA level in HSP27 overexpression group had 36 and 29\% reduction, compared with the control group. Data represent one of three independent experiments.

\section{DISCUSSION}

It is well known that mild temperature elevation can induce a rapid production of a special class of proteins called HSPs or chaperones. Now, it is known that other physical 
agents (Nussbaum and Locke, 2007) or drugs can also induce HSPs (Hu et al., 2011). HSPs comprise several families of proteins expressed by a number of cell types following exposure to stressful environmental conditions that include heat, free radicals, toxins, and ischemia. HSP27 is a member of the small HSP family with a molecular weight of approximately 27 $\mathrm{kDa}$. In addition to its chaperone functions, HSP27 also appears to be involved in a diverse range of cellular functions, promoting cell survival through effects on the apoptotic pathway and plays important roles in cytoskeleton dynamics and cell differentiation (GhayourMobarhan et al., 2012).

HSP27 plays an essential role in various diseases (Mullen et al., 2011), and can be a biomarker of disease and therapeutic target (Vidyasagar et al., 2012). Manipulation of upregulation of HSPs in cells has been demonstrated to provide a therapeutic strategy to counteract the misfolding and aggregation of proteins that result in neurodegenerative disease (Kong et al., 2011). The data showed that in a tg mouse model, overexpression of either wild-type hsp27 or a nonphosphorylatable hsp27 mutant was equally capable of protecting the heart from I/R injury (Hollander et al., 2004). Their function is thought to be the quick resolution of misfolded proteins and the development of thermotolerance. From this point of view, the production of HSPs has been considered to be beneficial for cells and tissues. Contradictory results have also been obtained for ultrasound treatment. For example, the treatment of mice with ultrasound (1 MHz) results in an increase in Hsp72 protein content (Smith et al., 2002), whereas after the treatment of rats with ultrasound of $1 \mathrm{MHz}$, there is no alteration in Hsp72 production (Locke and Nussbaum, 2001).

Low-frequency ultrasound $(20-100 \mathrm{kHz})$ has a diverse set of industrial and medical applications. In fact, high-power industrial applications of ultrasound mainly occupy this frequency range. This range is also used for various therapeutic medical applications including sonophoresis (ultrasonic transdermal drug delivery), dentistry, eye surgery, body contouring, breakage of kidney stones, and elimination of blood clots (Ahmadi et al., 2012).

A new noninvasive method for the detection of DNA damage using mid- to highfrequency ultrasound (10-60 MHz) has been developed for future investigations regarding the use of ultrasound in preclinical and clinical settings to noninvasively monitor tumor responses to specific interventions that induce cell death (Vlad et al., 2011). However, the few reports on aortic smooth muscular cells prompted us to investigate if there is any protection from cell death in HSP27-overexpressing HASMC exposed to LFLEU. An HSP27-overexpressing HASMC line was constructed for this purpose. Apoptosis in this cell line was evaluated at the optimal time point determined for the occurrence of cell death $(30 \mathrm{~s})$, where exposure to ultrasound alone resulted in minimal cell death as shown in Figure 3.

The evidence showed that ultrasound exposure of malignant lymphoid cell lines is associated with time-dependent apoptosis, which is mitochondrial-caspase dependent (Firestein et al., 2003). Apoptosis is activated by extrinsic (death ligand) and intrinsic (mitochondrial) pathways (Salvesen, 2002; Ghavami et al., 2009). This extrinsic activation then triggers the hallmark caspase cascade characteristic of the apoptotic pathway, in which caspase-3 plays a dominant role (Perry et al., 1997; Stennicke et al., 2000). In intrinsic activation, cytochrome c from the mitochondria works in combination with caspase-9, apoptosis-activating factor 1 (Apaf-1), and ATP to process procaspase-3 (Katunuma et al., 2001; Li et al., 2004). We found that the apoptosis rate was significantly lower in HSP27-overexpressing HASMC with 30 -s ultrasound exposure, compared with the control group. There was no significant change 
between control and vector group. After the exposure time (30 s), gene chip analysis showed downregulated caspase- 9 expression, which has been considered to be a protective mechanism for HASMC exposed to LFLEU. Although caspase-3 was upregulated according to gene chip analysis (Table 1), Western blot analysis and RT-PCR proved that it was downregulated (Figure 5), indicating that gene display analysis is not accurate due to higher background noise. From this point of view, our results supported that higher expression of HSP27 is beneficial for cells and tissues by inhibiting the mitochondrial caspase-dependent apoptotic pathway. Further research is needed to elucidate detailed mechanisms, such as how hsp27 can downregulate caspase- 9 expression and whether caspase- 8 is involved in the apoptosis, although it belongs to the extrinsic (death ligand) pathway.

So far, this is the first report that HSP27 overexpression in HASMC is capable of protecting against apoptosis induced by low-frequency ultrasound by downregulating caspase- 9 expression in intrinsic (mitochondrial) pathways.

\section{ACKNOWLEDGMENTS}

Research supported by the Shanghai Pujiang Project of China (Grant \#09PJ1408800), and Major Infrastructure Projects of Shanghai Science and Technology (Grant\#10JC1412600).

\section{Conflicts of interest}

The authors declare no conflict of interest.

\section{REFERENCES}

Ahmadi F, McLoughlin IV, Chauhan S and ter-Haar G (2012). Bio-effects and safety of low-intensity, low-frequency ultrasonic exposure. Prog. Biophys. Mol. Biol. 108: 119-138.

Arakawa K, Hagisawa K, Kusano H, Yoneyama S, et al. (2002). Sonodynamic therapy decreased neointimal hyperplasia after stenting in the rabbit iliac artery. Circulation 105: 149-151.

Baker ML and Dalrymple GV (1978). Biological effects of diagnostic ultrasound: a review. Radiology 126: 479-483.

Benjamin IJ and McMillan DR (1998). Stress (heat shock) proteins: molecular chaperones in cardiovascular biology and disease. Circ. Res. 83: 117-132.

Chelouche-Lev D, Kluger HM, Berger AJ, Rimm DL, et al. (2004). alphaB-crystallin as a marker of lymph node involvement in breast carcinoma. Cancer 100: 2543-2548.

Fang HY, Tsai KC, Cheng WH, Shieh MJ, et al. (2007). The effects of power on-off durations of pulsed ultrasound on the destruction of cancer cells. Int. J. Hyperther. 23: 371-380.

Feng Y, Tian ZM, Wan MX and Zheng ZB (2008). Low intensity ultrasound-induced apoptosis in human gastric carcinoma cells. World J. Gastroenterol. 14: 4873-4879.

Firestein F, Rozenszajn LA, Shemesh-Darvish L, Elimelech R, et al. (2003). Induction of apoptosis by ultrasound application in human malignant lymphoid cells: role of mitochondria-caspase pathway activation. Ann. N.Y. Acad. Sci. 1010: 163-166.

Ghavami S, Hashemi M, Ande SR, Yeganeh B, et al. (2009). Apoptosis and cancer: mutations within caspase genes. $J$. Med. Genet. 46: 497-510.

Ghayour-Mobarhan M, Saber H and Ferns GA (2012). The potential role of heat shock protein 27 in cardiovascular disease. Clin. Chim. Acta 413: 15-24.

Hendrick JP and Hartl FU (1993). Molecular chaperone functions of heat-shock proteins. Annu. Rev. Biochem. 62: 349-384.

Hollander JM, Martin JL, Belke DD, Scott BT, et al. (2004). Overexpression of wild-type heat shock protein 27 and a nonphosphorylatable heat shock protein 27 mutant protects against ischemia/reperfusion injury in a transgenic mouse model. Circulation 110: 3544-3552. 
Hu H, Wang L, Okauchi M, Keep RF, et al. (2011). Deferoxamine affects heat shock protein expression in heart after intracerebral hemorrhage in aged rats. Acta Neurochir. Suppl. 111: 197-200.

Kampinga HH, Hageman J, Vos MJ, Kubota H, et al. (2009). Guidelines for the nomenclature of the human heat shock proteins. Cell Stress Chaperones 14: 105-111.

Katunuma N, Matsui A, Le QT, Utsumi K, et al. (2001). Novel procaspase-3 activating cascade mediated by lysoapoptases and its biological significances in apoptosis. Adv. Enzyme Regul. 41: 237-250.

Kong XC, Zhang D, Qian C, Liu GT, et al. (2011). FLZ, a novel HSP27 and HSP70 inducer, protects SH-SY5Y cells from apoptosis caused by MPP(+). Brain Res. 1383: 99-107.

Lagneaux L, de Meulenaer EC, Delforge A, Dejeneffe M, et al. (2002). Ultrasonic low-energy treatment: a novel approach to induce apoptosis in human leukemic cells. Exp. Hematol. 30: 1293-1301.

Li P, Nijhawan D and Wang X (2004). Mitochondrial activation of apoptosis. Cell 116: S57-9, 2.

Liu AY, Lin Z, Choi HS, Sorhage F, et al. (1989). Attenuated induction of heat shock gene expression in aging diploid fibroblasts. J. Biol. Chem. 264: 12037-12045.

Locke M and Nussbaum E (2001). Continuous and pulsed ultrasound do not increase heat shock protein 72 content. Ultrasound Med. Biol. 27: 1413-1419.

Mackay DS, Andley UP and Shiels A (2003). Cell death triggered by a novel mutation in the alphaA-crystallin gene underlies autosomal dominant cataract linked to chromosome 21q. Eur. J. Hum. Genet. 11: 784-793.

Man J, Shelton RM, Cooper PR and Scheven BA (2012). Low-intensity low-frequency ultrasound promotes proliferation and differentiation of odontoblast-like cells. J. Endod. 38: 608-613.

Martin JL, Mestril R, Hilal-Dandan R, Brunton LL, et al. (1997). Small heat shock proteins and protection against ischemic injury in cardiac myocytes. Circulation 96: 4343-4348.

Meehan S, Berry Y, Luisi B, Dobson CM, et al. (2004). Amyloid fibril formation by lens crystallin proteins and its implications for cataract formation. J. Biol. Chem. 279: 3413-3419.

Mullen E, O'Reilly E and Ohlendieck K (2011). Skeletal muscle tissue from the Goto-Kakizaki rat model of type-2 diabetes exhibits increased levels of the small heat shock protein Hsp27. Mol. Med. Rep. 4: 229-236.

Nussbaum EL and Locke M (2007). Heat shock protein expression in rat skeletal muscle after repeated applications of pulsed and continuous ultrasound. Arch. Phys. Med. Rehabil. 88: 785-790.

Palumbo P, Cinque B, Miconi G, La Torre C, et al. (2011). Biological effects of low frequency high intensity ultrasound application on ex vivo human adipose tissue. Int. J. Immunopathol. Pharmacol. 24: 411-422.

Perry DK, Smyth MJ, Stennicke HR, Salvesen GS, et al. (1997). Zinc is a potent inhibitor of the apoptotic protease, caspase-3. A novel target for zinc in the inhibition of apoptosis. J. Biol. Chem. 272: 18530-18533.

Reimers B, Moussa I, Akiyama T, Tucci G, et al. (1997). Long-term clinical follow-up after successful repeat percutaneous intervention for stent restenosis. J. Am. Coll. Cardiol. 30: 186-192.

Rokhina EV, Lens P and Virkutyte J (2009). Low-frequency ultrasound in biotechnology: state of the art. Trends Biotechnol. 27: 298-306.

Salinthone S, Tyagi M and Gerthoffer WT (2008). Small heat shock proteins in smooth muscle. Pharmacol. Ther. 119: 44-54.

Salvesen GS (2002). Caspases: opening the boxes and interpreting the arrows. Cell Death Differ. 9: 3-5.

Smith RC, Machluf M, Bromley P, Atala A, et al. (2002). Spatial and temporal control of transgene expression through ultrasound-mediated induction of the heat shock protein 70B promoter in vivo. Hum. Gene Ther. 13: 697-706.

Stennicke HR, Renatus M, Meldal M and Salvesen GS (2000). Internally quenched fluorescent peptide substrates disclose the subsite preferences of human caspases 1, 3, 6, 7 and 8. Biochem. J. 350 (Pt 2): 563-568.

Taylor RP and Benjamin IJ (2005). Small heat shock proteins: a new classification scheme in mammals. J. Mol. Cell Cardiol. 38: 433-444.

Tsuang YH, Liao LW, Chao YH, Sun JS, et al. (2011). Effects of low intensity pulsed ultrasound on rat Schwann cells metabolism. Artif. Organs 35: 373-383.

van Noort JM, van Sechel AC, Bajramovic JJ, el Ouagmiri M, et al. (1995). The small heat-shock protein alpha B-crystallin as candidate autoantigen in multiple sclerosis. Nature 375: 798-801.

Vidyasagar A, Wilson NA and Djamali A (2012). Heat shock protein 27 (HSP27): biomarker of disease and therapeutic target. Fibrogenesis Tissue Repair 5: 7.

Vlad RM, Kolios MC and Czarnota GJ (2011). Ultrasound imaging of apoptosis: spectroscopic detection of DNA-damage effects at high and low frequencies. Methods Mol. Biol. 682: 165-187.

Xia CY, Liu YH, Wang P and Xue YX (2012). Low-frequency ultrasound irradiation increases blood-tumor barrier permeability by transcellular pathway in a rat glioma model. J. Mol. Neurosci. 48: 281-290. 